\title{
Anatomical considerations of the infrapyloric artery and its associated lymph nodes during laparoscopic gastric cancer surgery
}

\author{
Shusuke Haruta $\cdot$ Hisashi Shinohara $\cdot$ \\ Masaki Ueno · Harushi Udagawa · Yoshiharu Sakai • \\ Ichiro Uyama
}

Received: 27 May 2014/ Accepted: 23 August 2014/Published online: 17 September 2014

(C) The International Gastric Cancer Association and The Japanese Gastric Cancer Association 2014

\begin{abstract}
Background Little is known about the vascular and lymphatic distribution of the pyloric antrum in the stomach. We focused on the infrapyloric region containing the infrapyloric artery (IPA) and lymph nodes.

Methods The anatomy of the IPA and its associated lymph nodes was clinically elucidated during 156 laparoscopic gastrectomies.

Results Most of the arteries originated from the anterior superior pancreatoduodenal artery (ASPDA, $64.2 \%$ ) or the root of the right gastroepiploic artery (RGEA, $23.1 \%$ ), but a small portion originated from the gastroduodenal artery (GDA, $12.7 \%$ ). The average lengths from the pyloric ring to the IPA proximal branch were $21.8 \mathrm{~mm}$ from the ASPDA, $20.6 \mathrm{~mm}$ from the RGEA and $9.0 \mathrm{~mm}$ from the GDA, a significantly shorter length than the other 2 variations. On average, 2.5 out of 10.0 nodes existed along the IPA. One patient, whose tumor was located close to the pylorus, had a metastatic node in this section.

Conclusion The IPA most commonly originates from the ASPDA and is associated with a certain number of lymph nodes. Vascular distribution from the IPA depends on the anatomic variation.
\end{abstract}

S. Haruta and H. Shinohara contributed equally to this work.

S. Haruta $\cdot$ H. Shinohara $(\bowtie) \cdot$ M. Ueno $\cdot$ H. Udagawa

Department of Gastroenterological Surgery, Toranomon

Hospital, 2-2-2 Toranomon, Minato-ku, Tokyo 105-8470, Japan

e-mail: shinohara@toranomon.gr.jp

S. Haruta · I. Uyama

Department of Surgery, Fujita Health University, Aichi, Japan

Y. Sakai

Department of Surgery, Kyoto University Graduate School of Medicine, Kyoto, Japan
Keywords Infrapyloric artery · Station no.6 · Lymphadenectomy · Laparoscopic surgery · Gastric cancer

\section{Introduction}

The lymph nodes (LNs) lying in the infrapyloric area, categorized as the no. 6 by the Japanese Gastric Cancer Association (JGCA) [1], is considered an important confluence of lymphatic channels that drain the distal twothirds of the stomach [2-4]. Several studies have demonstrated that metastasis to the no. 6 nodes is very common; thereby making the dissection of this station an important surgical step [5, 6]. JGCA has defined the no. 6 station as the area along the proximal part of the right gastroepiploic artery (RGEA) and vein [1]. That may make sense because most of lymphatic channels are accompanied by feeding vasculatures. However, blood flow to the pyloric antrum is predominantly supplied by the infrapyloric artery (IPA) [7, 8]. If the IPA is independent of the RGEA and is associated with regional LNs, anatomical knowledge of this artery will be required not only for the pylorus preserving procedures but also for the reasonable dissection of the infrapyloric region.

Despite previous angiography and cadaver autopsy studies, the anatomy of the IPA is poorly understood because it is too small for precise examination [9-13]. Recent advances in laparoscopic techniques, however, have provided surgeons with magnified and highly defined images [14-17]. In the present study, we elucidated the anatomy of the IPA by precisely identifying and dividing this artery during no. 6 lymphadenectomy. Also, the distribution of IPA tributaries to the pylorus and the number and metastatic status of LNs situated along the artery were investigated using surgical specimens. 


\section{Methods}

Patients

This study included 156 patients who underwent laparoscopic gastrectomy for early gastric cancer, clinically estimated to be $\mathrm{T} 1$ in depth. The study was conducted between December 2011 and April 2014 at Toranomon Hospital in Tokyo, Japan. Total gastrectomies were performed in 29 patients and distal gastrectomies were performed in 127 patients. None of the patients underwent a pylorus-preserving gastrectomy (PPG); therefore, standard no. 6 lymphadenectomies were performed in all cases.

Identification and division of IPA during no. 6 lymphadenectomies

The surgical setting of our laparoscopic gastrectomies and our procedure for the no. $6 \mathrm{LN}$ dissection has been described previously [2]. After division of the greater omentum, the transverse smesocolon was taken down to identify the confluence of the right gastroepiploic vein (RGEV) and the anterior superior pancreatoduodenal vein (ASPDV). Subsequently, the RGEV and the infrapyloric vein (IPV) were ligated with clips and the adipose tissue, with its included LNs, was carefully dissected from the anterior surface of the head of the pancreas.

Next, we detached the pancreatic parenchyma from the posterior wall of the duodenal bulb to expose the gastroduodenal artery (GDA). The RGEA root was isolated by dissecting away the surrounding adipose tissue and nerve fibers. At this point, we were usually able to decipher whether the IPA was branched from the distal side (ASPDA) or proximal side (GDA) of the RGEA, except in cases when the IPA branched from the caudal part of the RGEA. The RGEA was ligated with double clips at its root and then the IPA was subsequently ligated. Finally, the inferior wall of the duodenal bulb was skeletonized, and the no. 6 nodal region was removed en bloc with the gastric specimen.

Investigation of IPA distribution to the pyloric antrum

Following resection, the surgical specimen was submitted for investigation to determine the morphometric anatomy of blood vessels. The distance from the pyloric ring to the first branch off of the RGEA that supplies the greater curvature was measured. Additionally, vascular distribution from the IPA was examined by measuring the length from the pyloric ring to the first proximal branch point along the IPA.

Examination of no. 6 lymph nodes

The soft tissue, including the no. 6 nodes, was then divided into three sections, according to previously described

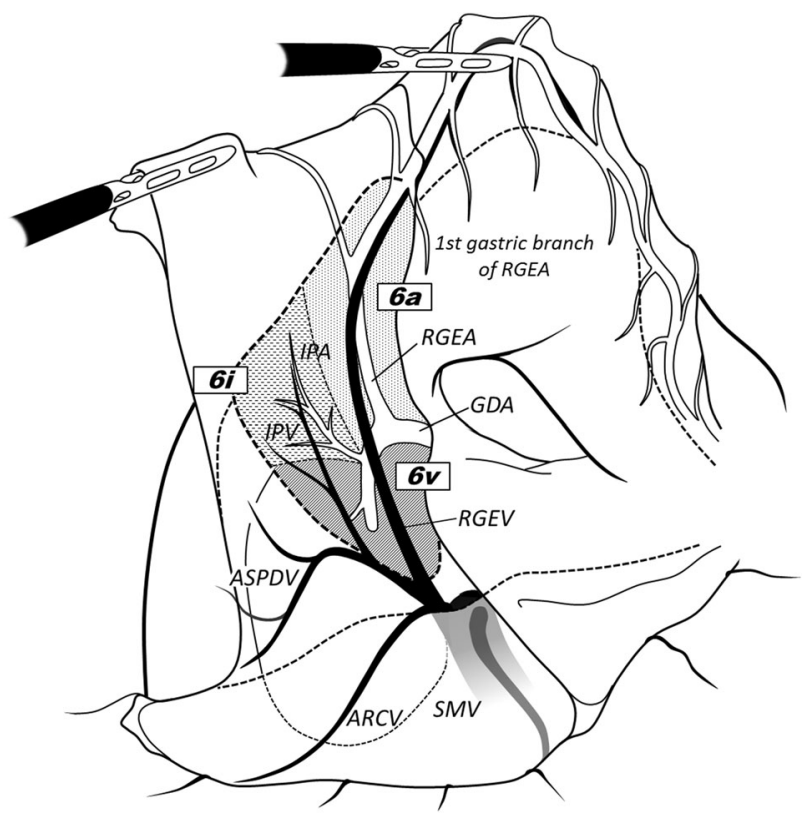

Fig. 1 The representative vascular anatomy of the infrapyloric lymph node station no. 6 that has been divided into three sections. These sections were divided along the proximal part of the right gastroepiploic vein (RGEV, 6v), the right gastroepiploic artery (RGEA, 6a), and the infrapyloric artery (IPA, 6i). GDA gastroduodenal artery, $A S P D V$ anterior superior pancreatoduodenal vein, $I P V$ infrapyloric vein, $S M V$ superior mesenteric vein, $A R C V$ accessory right colic vein, $G C T$ gastrocolic trunk. This figure was quoted from Ref. [2] with some modifications under the permission of the authors

criteria [2]. In short; section 6a is adjacent to the RGEA from its first gastric branch to its root, section $6 \mathrm{v}$ is adjacent to the RGEV, between the root of the RGEA and the confluence of the ASPDV, and section 6i is located adjacent to the IPA (Fig. 1). The section 6i was discriminated from the section 6a along the avascular area between the proximal branch of the IPA and the RGEA. LNs from each section were then harvested and prepared for histological examination.

Statistics

Statistical analysis was performed using the unpaired Student's $t$ test or the Chi square test. A $P$-value of less than 0.05 was regarded as statistically significant. All values henceforth are expressed in the following manner: mean (standard deviation).

\section{Results}

Origin of the IPA

The IPA was located and ligated intraoperatively in 115 cases and detected postoperatively in soft tissue specimens 


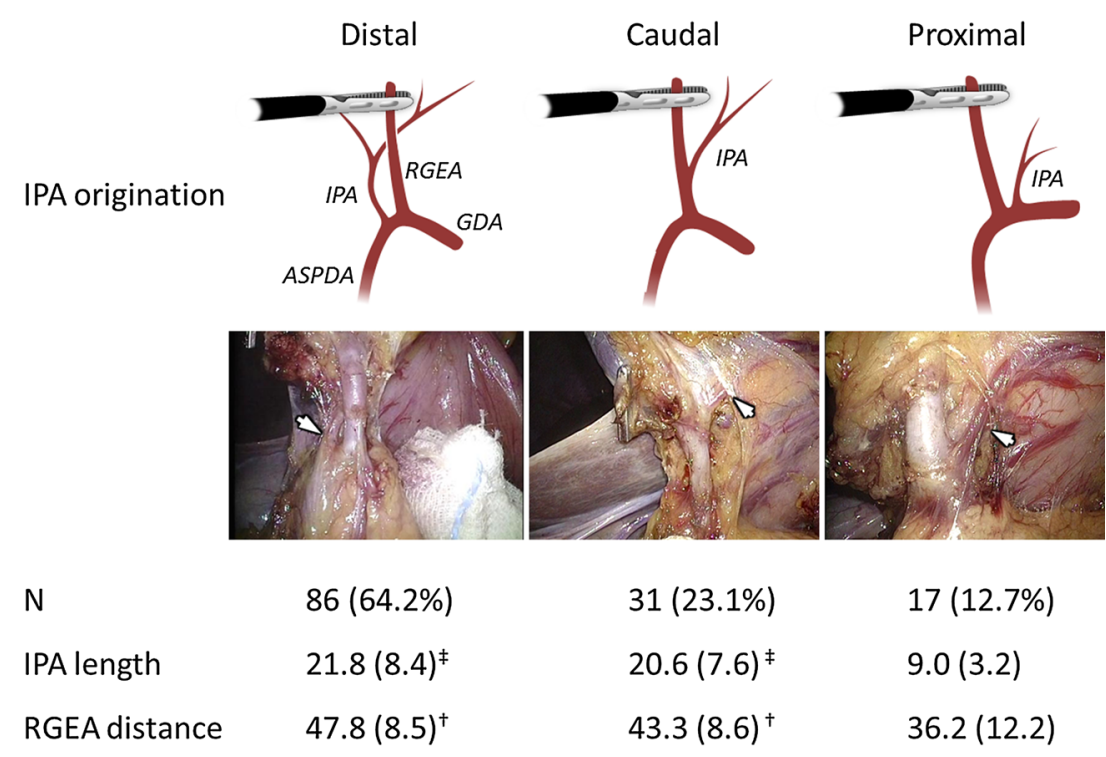

Fig. 2 The origin of the infrapyloric artery (IPA) has been classified into three types: distal type, in which the IPA originates from the anterior superior pancreatoduodenal artery (ASPDA), caudal type, in which the IPA originates from the right gastroepiploic artery (RGEA), and proximal type, in which the IPA originates from the gastroduodenal artery (GDA). The arrows in the pictures indicate the origin of

in the remaining 41 cases. In the latter set of cases, the origin of the IPA was identified in 19 out of 41 specimens. Therefore, a total of 134 samples were eligible for this study. The arteries from which the IPA originated were classified into three types: distal type (with the IPA originating from the ASPDA), proximal type (from the GDA), and caudal type (from the RGEA) (Fig. 2). Eighty-six cases $(64.2 \%)$ were categorized as distal type, 17 cases $(12.7 \%)$ were categorized as proximal type, and 31 cases (23.1\%) including all of the 19 cases in which the origin IPA was detected in the specimens after surgery were categorized as caudal type (Fig. 2). The branching points in 28 out of 86 distal type cases and 7 out of 17 proximal type cases were, however, very close to the root of the RGEA.

\section{Vascular distribution from the IPA}

We examined the distribution of the IPA tributaries in the surgical specimens. Results and representative pictures of distal and caudal types are summarized in Fig. 2 and shown in Fig. 3, respectively. The lengths from the pyloric ring to the proximal branch of the IPA were $21.8 \mathrm{~mm}(8.4)$ and $20.6 \mathrm{~mm}$ (7.6) in distal and caudal types, respectively. These were significantly longer than the average value of the proximal type, which was $9.0 \mathrm{~mm}$ (3.2) in length. The distances from the pyloric ring to the first gastric branch of the RGEA, which corresponds to the width of no. 6 area, were $47.8 \mathrm{~mm}$ (8.5) for the distal type and $43.3 \mathrm{~mm}(8.6)$ for caudal type. Similarly, these were significantly longer the IPA. IPA length represents the length from the pyloric ring to the proximal branch of the IPA (mm); RGEA distance represents the distance from the pyloric ring to the first gastric branch of the RGEA $(\mathrm{mm})$. Values in parentheses represent the relative standard deviations. ${ }^{\ddagger} P<0.01,{ }^{\dagger} P<0.05$ compared with Proximal type

than the value of the proximal type, which was $36.2 \mathrm{~mm}$ (12.2) in width.

Lymph nodes in the infrapyloric area

Finally, we examined how many LNs existed along the IPA. On average, 5.0 (3.2), 2.5 (2.0) and 2.5 (2.1) LNs were harvested from the sections $6 \mathrm{a}, 6 \mathrm{v}$ and $6 \mathrm{i}$, respectively. Metastases to no. 6 were found in 5 out of 156 patients. Four patients had a metastatic LN in section $6 \mathrm{a}$ and 1 patient had three metastatic nodes in section $6 \mathrm{v}$. One patient, who had a $\mathrm{T} 1$ tumor of the antrum, $2 \mathrm{~cm}$ proximal to the pylorus, had a metastatic node in section $6 \mathrm{i}$.

\section{Discussion}

Among the regional LNs of the stomach, the no. 6 infrapyloric nodes lie in an anatomically important area. The JGCA has defined the "afferent section" as the area along the first branch and the proximal section of the RGEA; the "efferent section" is defined as the area from the border of the afferent section to the confluence of the RGEV and the ASPDV [1]. Blood flow from the pyloric antrum, however, is predominantly supplied by its own artery, the IPA [9]. Thus, we focused on the anatomy of this artery and its associated LNs.

The IPA, a small artery whose diameter is approximately $1.2 \mathrm{~mm}$ [9], was initially described in 1904 as 


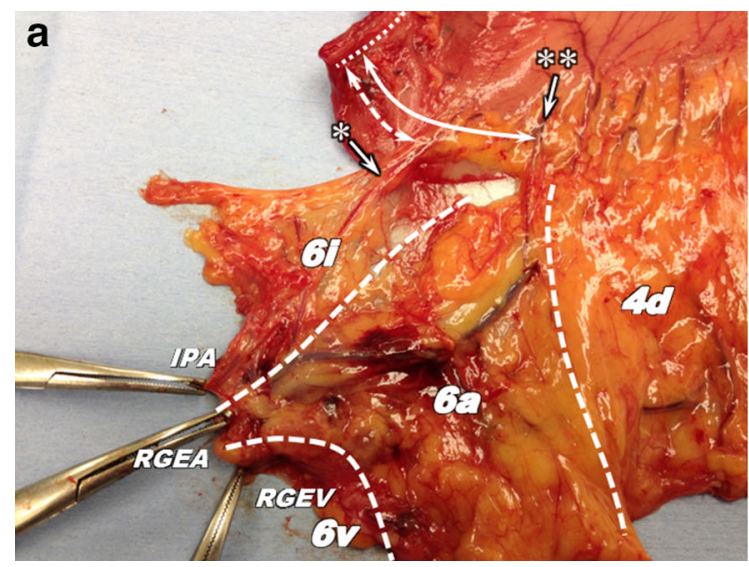

Fig. 3 Representative distal-type a and caudal-type b surgical specimens, demonstrating the infrapyloric lymph station no. 6 vasculature divided into three sections. Dotted lines indicate the pyloric ring, dashed arrows indicate the length from the pyloric ring to the

"Ciuffo pilorico inferior" by Rossi et al. [10], and identified as the "pyloric branch" in 1982 by Kuroda et al. [11]. The term "infrapyloric artery" was first cited in 1988, referring to the IPA originating from the RGEA [12]. Studies evaluating the origin of the IPA, however, vary throughout the literature. Sawai et al. [13] reported that the IPA originates from the GDA in $64 \%$, from the ASPDA in $21 \%$, and from the RGEA in only $12 \%$ of gastric cancer patients, determined via angiography. In contrast, a recent study by Takamuro et al. [9] using 214 cadavers confirmed that nearly $50 \%$ of IPAs originate from the ASPDA or from the wedged position, between the ASPDA and RGEA. In the present study, which included 134 early gastric cancer surgeries, we have carefully explored the origins of the IPA around the root of the RGEA with precise laparoscopic images. Our results demonstrate that most of the IPAs originated from the previously defined distal or caudal site; however only $12.7 \%$ originated proximal to the root of the RGEA. These data support the findings obtained from the cadaver study by Takamuro et al. [9]. Discrepancies from previous reports may be due to the difficulty in identifying these arteries because of their small diameters.

The average number of LNs harvested from section $6 \mathrm{i}$ (along the IPA) was 2.5. Although we did not detect lymphatic vessels, our data suggest the existence of afferent lymphatic fluid that flows alongside the IPA from the pyloric antrum to the root of the RGEA. In the present study, one patient whose tumor was located in the antrum fed by the IPA had metastases to this section. Conversely, it may be possible to exclude the section $6 \mathrm{i}$ dissection in patients undergoing a PPG with an indication directly based on tumor location and depth of invasion.

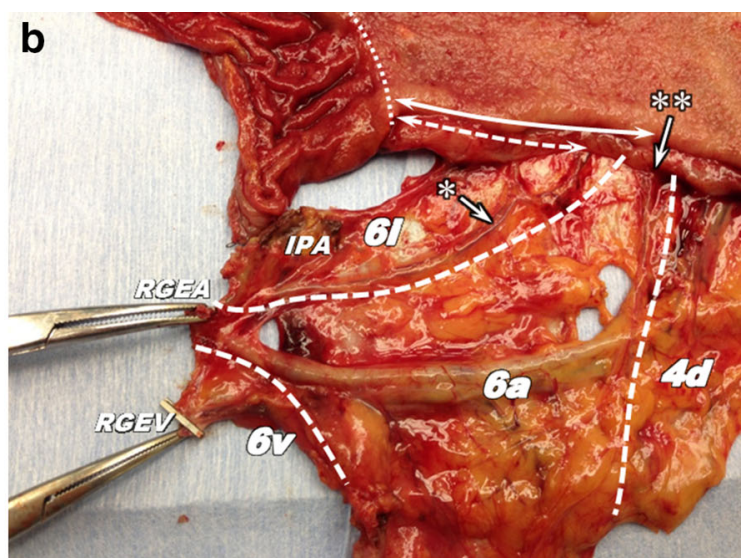

proximal branch of the infrapyloric artery (IPA, asterisk), and solid arrows indicate the distance from the pyloric ring to the first gastric branch of the right gastroepiploic artery (RGEA, double asterisk). $R G E V$ right gastroepiploic vein

In conclusion, the IPA predominantly feeds the pyloric antrum, usually originates from the ASPDA and is accompanied by a certain number of LNs. Consideration of the findings of this study will provide for safer, more oncologically feasible lymph node dissection of the infrapyloric region in gastric cancer surgery.

Acknowledgments We thank Dr. Kyle J. Litow for English proofreading.

\section{References}

1. Japanese Gastric Cancer Association. Japanese classification of gastric carcinoma: 3rd English edition. Gastric Cancer. 2011;14:101-12.

2. Shinohara H, Kurahashi Y, Kanaya S, Haruta S, Ueno M, Udagawa $\mathrm{H}$, et al. Topographic anatomy and laparoscopic technique for dissection of no. 6 infrapyloric lymph nodes in gastric surgery. Gastric Cancer. 2013;16(4):615-20.

3. Pólya E, von Navratil D. Untersuchung über die Lymphbahnen des Wurmfortsatzes und des Magens. Zeitschr für klin Chir. 1903;69:421-56.

4. Pólya E. Zur Stumpfversorgung nach Magenresektion. Zentrbl für Chir. 1911;38:892-4.

5. Sasako M, McCulloch P, Kinoshita T, Maruyama K. New method to evaluate the therapeutic value of lymph node dissection for gastric cancer. Br J Surg. 1995;82:346-51.

6. Maruyama K, Gunvēn P, Okabayashi K, Sasako M, Kinoshita T. Lymph node metastases of gastric cancer. General pattern in General pattern in 1931 patients. Ann Surg. 1931;1989(210):596-602.

7. Liberski SM, Koch KL, Atnip RG, Stern RM. Ischemic gastroparesis: resolution after revascularization. Gastroenterology. 1990;99:252-7.

8. Wilkie D. Blood supply of the duodenum. Surg Gynecol Obstet. 1911;3:399-405.

9. Takamuro T, Murakami G, Hirata K. Arterial supply of the first, third and fourth portion of the duodenum: an anatomical study with special reference to the minimal invasive 
pancreaticoduodenectomy (in Japanese). Jpn J Gastroenterol Surg. 1998;31:825-35.

10. Rossi G, Cova E. Studio morfolgico delle arterie dello stomaco. Arch Ital Anat Embriol. 1904;3:485.

11. Kuroda C, Nakamura H, Sato T, Yoshioka H, Tokunaga K, Hori $\mathrm{S}$, et al. Normal anatomy of the pyrolic branch and its diagnostic significance in angiography. Acta Radiol Diagn. 1982;23:479-84.

12. Vandamme J, Bonte J. The blood supply of the stomach. Acta Anat. 1988;131:89-96.

13. Sawai K, Takahashi T, Fujioka $T$, Minato $H$, Tanigushi $H$, Yamagushi T. Pylorus-preserving gastrectomy with radical lymph node dissection based on anatomical variations of the infrapyloric artery. Am J Surg. 1995;170:285-8.

14. Uyama I, Sugioka A, Matsui H, Fujita J, Komori Y, Hasumi A. Laparoscopic D2 lymph node dissection for advanced gastric cancer located in the middle or lower third portion of the stomach. Gastric Cancer. 2000;3:50-5.
15. Kanaya $S$, Haruta $S$, Kawamura $Y$, Yoshimura F, Inaba K, Hiramatsu $\mathrm{Y}$, et al. Laparoscopy distinctive technique for suprapancreatic lymph node dissection: medial approach for laparoscopic gastric cancer surgery. Surg Endosc. 2011;25: 3928-9.

16. Obama K, Okabe H, Hosogi H, Tanaka E, Itami A, Sakai Y. Feasibility of laparoscopic gastrectomy with radical lymph node dissection for gastric cancer: from a viewpoint of pancreas-related complications. Surgery. 2011;149:15-21.

17. Noshiro H, Nagai E, Shimizu S, Uchiyama A, Tanaka M. Laparoscopically assisted distal gastrectomy with standard radical lymph node dissection for gastric cancer. Surg Endosc. 2005;19:1592-6. 